\title{
POST-PRANDIAL ELECTROCARDIOGRAPHIC CHANGES BEFORE AND AFTER GASTRECTOMY
}

\author{
BY \\ J. W. KERR* AND H. L. DUTHIE $\dagger$ \\ From the Western Infirmary, Glasgow
}

Received June 12, 1961

Changes in the electrocardiogram after a meal in normal patients have been described by several authors since Gardberg and Olsen (1939) showed a 30-50 per cent decrease in the height of the T wave thirty minutes after a meal, a change which lasted up to two hours. A statistical study of the effect of meals on patients was carried out by Simonson et al. (1946) and further amplified by Simonson and Keys (1950). They believed that the $T$ wave changes might be explained by sympathetic stimulation, and did not think diminished coronary flow was the cause. They were of the opinion that the $T$ wave changes were associated with a change in the ventricular gradient, and this view was supported by Rosen and Gardberg $(1957 a, b)$. The use of a glucose meal (100 g.) elicited E.C.G. changes of a similar pattern in 47 out of 85 normal patients (Rochlin and Edwards, 1954).

Subtotal gastric resection is still one of the main methods of surgical treatment of peptic ulcer. Although this operation removes ulcer symptoms there can be disappointing post-prandial symptoms often associated with E.C.G. changes. In the early post-operative weeks, most patients complain of fulness in the epigastrium which may amount to discomfort. A minority have, in addition, faintness, tiredness, palpitations, sweating, pallor or subjective flushing, and sometimes nausea and bilious vomiting. This symptom complex, usually known as the dumping syndrome (Mix, 1922), may last for years, though many patients recover spontaneously within the first few months following operation. These symptoms begin 15-20 minutes after eating and last up to one hour. Relief is usually obtained by lying down. Those patients whose symptoms appeared soon after a meal were said to have the early post-gastrectomy or dumping syndrome (Adlersberg and Hammerschlag, 1947; Zollinger and Hoerr, 1947), while the rarer group with hypoglycæmic symptoms two to three hours after eating were said to have the late post-gastrectomy syndrome. Electrocardiographic changes coincide with the early post-gastrectomy syndrome.

Changes in the E.C.G. after a meal in patients who have had a gastrectomy have been previously reported (Smith, 1951; Smith et al., 1953; Pulvertaft, 1954; Roberts et al., 1954; Peddie et al., 1957; Duthie et al., 1959). It has not been suggested that these changes cause dumping but that they are caused by the same factors as the symptoms.

Four factors have been suggested as contributing to the E.C.G. changes following a meal in patients after gastrectomy, namely potassium deficiency, rapid glucose absorption, diminished coronary blood flow, and finally sympathetic overactivity. A fall in the serum potassium during dumping attacks was noted along with the E.C.G. and myographic changes suggestive of impaired muscle function (Smith, 1951), and the intravenous administration of potassium reversed the E.C.G. changes, prevented muscle weakness, and shortened the attacks. Further work (Smith et al., 1953) suggested that the rapid absorption of sugar and release of adrenaline might contribute to the

* Clinical Chemotherapy Research Unit of the Medical Research Council, Western Infirmary, Glasgow.

$\uparrow$ Department of Surgery, Western Infirmary, Glasgow; now at Department of Surgery, General Infirmary, Leeds. 
fall in serum potassium and thus to the E.C.G. changes. Roberts et al. (1954) could find no correlation between the symptoms and lowered levels of serum potassium. Pulvertaft (1954) found the symptoms occurred before the hypokalæmia and postulated a connection between the rate of absorption of sugar and the vasomotor symptoms and the E.C.G. changes.

In recent years attention has been given to circulatory changes. A decrease in blood volume has been observed at the time of symptoms induced by a glucose meal in patients after gastrectomy (Roberts et al., 1954; Roberts et al., 1955; Walker et al., 1955) together with E.C.G. changes similar to those reported by Smith (1951). Hypotension accompanied these findings in most cases, and it was suggested that the fall in blood pressure might result in stimulation of the sympathetic nervous system from baro-receptors in the arch of the aorta, the E.C.G. changes being explained on the basis of coronary insufficiency.

The first part of this paper reports the E.C.G. changes following a glucose meal in patients with intact stomach and after gastrectomy. During the test period, other cardiovascular parameters were observed and have been previously reported in part (Duthie et al., 1959). The second part consists of experiments carried out on patients with gastrectomy to evaluate the suggested mechanism of the E.C.G. changes.

\section{MATERIAL AND Methods}

Twelve patients were studied before operation, 36 after Polya gastrectomy (gastro-jejunal anastomosis), seven after gastro-jejunostomy with vagotomy, and five after conversion from Polya to Billroth I gastrectomy (gastro-duodenal anastomosis).

The patients were studied after a 12 hour fast, sitting in a chair with a control period of one hour before, and a test hour after the ingestion of $100 \mathrm{ml}$. of 50 per cent glucose. The E.C.G.s were taken during a two hour period during which the serum potassium, blood sugar level, plasma volume, and blood pressure were measured. Electrocardiograms were taken every few minutes with a Cambridge Electrite machine, recording the standard and augmented limb leads. The electrodes were not moved during the two hours, and to avoid postural effects on the E.C.G. the patient remained seated throughout the period of the test. The machine was standardized at each tracing to show a deflection of $1 \mathrm{~cm}$. per 1 millivolt. The QT ratio was taken as the relationship of the measured Q-T to the ideal Q-T, as calculated by Bazett's formula, expressed as a percentage (Goldberger, 1953).

Control studies were made over a period of two hours on patients who were not given a test meal, and no E.C.G. changes were observed. Since the ingestion of cold water can give rise to E.C.G. changes (Wilson and Finch, 1923), five patients were given $200 \mathrm{ml}$. of water at room temperature by mouth; but there was no alteration in the E.C.G. This indicated that any change following a glucose meal given at room temperature was not due to a cooling effect. It is generally agreed that subjective influences can play a part in the interpretation of E.C.G.s; care has therefore been taken throughout this study to use the patient as his own control.

\section{RESULTS}

Form of E.C.G. Changes. The changes were as follows in order of frequency (Table I, and Fig 1):

(i) An increase in the heart rate varying from a few beats to 50 beats a minute.

(ii) A broadening and lowering of the potential of the T wave best seen in leads II, III, and aVF; less frequent and less obvious in lead I. Accompanying this there was often a reciprocal decrease in the negativity of the $T$ wave in lead aVR. The Q-T ratio was increased.

(iii) An increase in the potential of the $P$ wave with peaking in leads II, III, and aVF.

(iv) A U wave made its appearance or became more prominent if already present.

(v) The S-T segment take-off was below the isoelectric line or the whole S-T segment became negative.

(vi) The QRS complex increased in voltage. 
TABLE I

Details of Changes in the Electrocardiogram Following a Glucose Meal

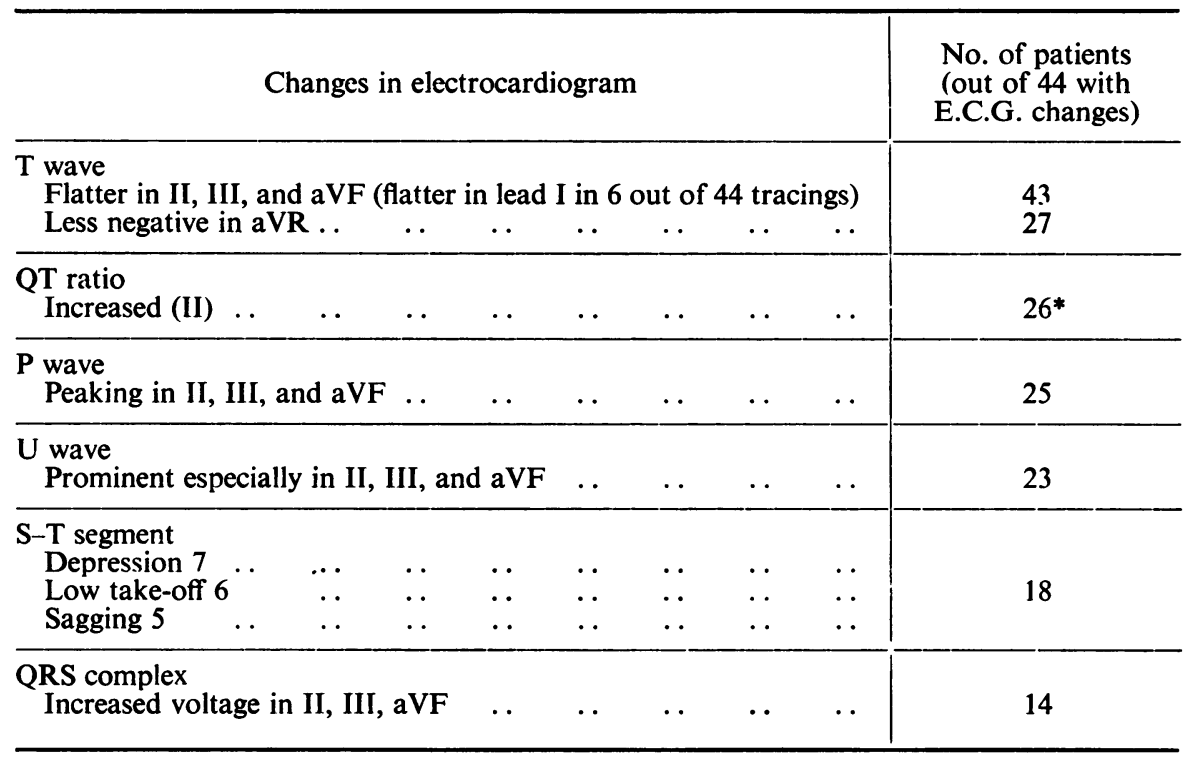

* Out of 31 patients with E.C.G. changes after Polya gastrectomy.

These changes appeared five to 15 minutes after the glucose meal, and usually reached a maximum at about 20 to 30 minutes. Thereafter they reverted slowly towards the control state, though residual changes were often present at the end of the test hour. Not all the above changes were seen in each case. The first change was flattening of the $T$ wave. The changes were reproducible as shown by repeat tests on the same patient.

Incidence of E.C.G. Changes. The changes occurred more often after operation than before, and particularly after the Polya type gastrectomy (Table II). Of the three patients with E.C.G.

TABLE II

The Incidence of Electrocardiographic Changes after a Standard Glucose Meal in Patients Before and AFTER OPERATION

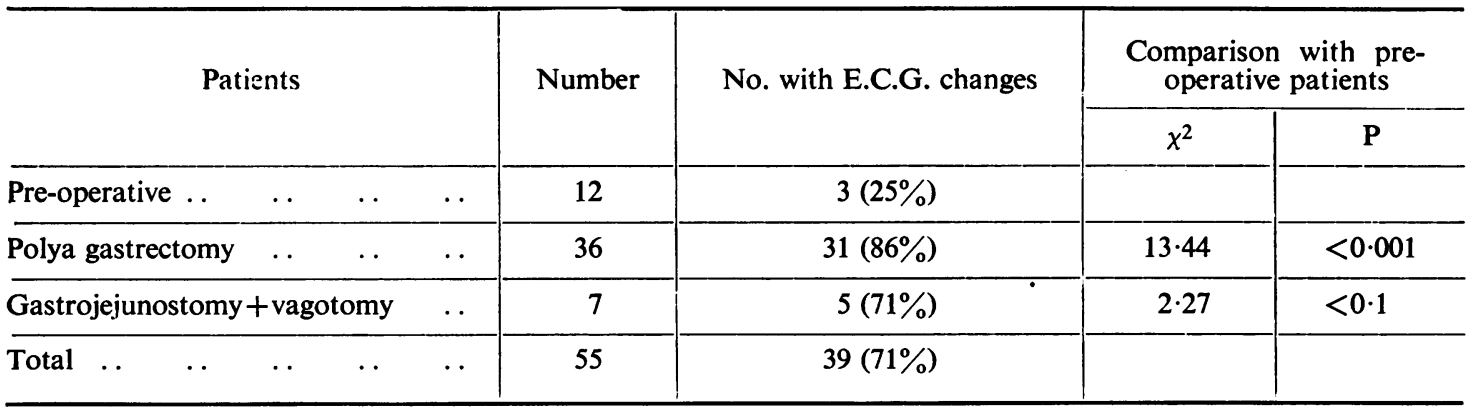

changes before operation, one exhibited slight $\mathrm{T}$ wave changes, and the other two had the full pattern. These two had similar changes after a Polya gastrectomy, though they appeared a little sooner after the meal. Neither had dumping symptoms. The E.C.G. changes were well marked in 26 of the 36 patients after Polya gastrectomy; five others had T wave changes only. 


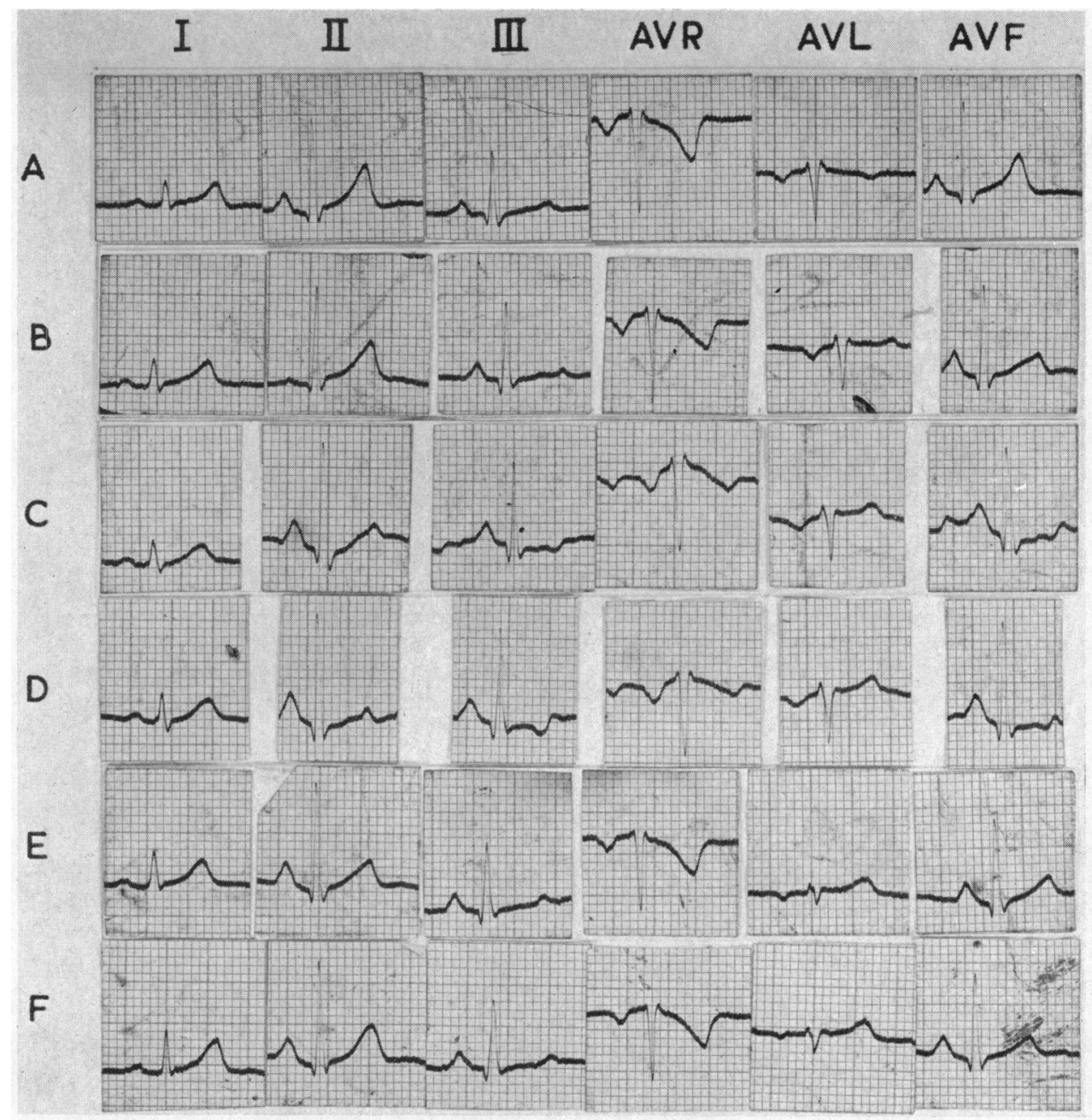

FIG. 1.-Electrocardiographic changes following a glucose meal in a patient after a Polya gastrectomy. A, control. B, C, D, and E, tracings taken at $6,12,25,40$, and 60 minutes respectively. Note the maximal changes present at 25 minutes.

The five patients who were studied after conversion to a Billroth I gastrectomy showed a pattern similar to that observed when they had a Polya anastomosis.

No correlation was found between the E.C.G. changes and change in blood pressure, pulse rate, or the degree of the fall in plasma volume. Only 17 of the 31 patients with E.C.G. changes after Polya gastrectomy had any form of dumping symptoms, and one had dumping symptoms without E.C.G. changes. Thus the presence of dumping symptoms was not necessarily associated with the E.C.G. changes.

Conclusion. It is clear that the E.C.G. changes following a glucose meal in patients after gastrectomy have the same pattern as those in a normal subject except that the changes are more frequent, occur more rapidly, and are more marked after the operation. 


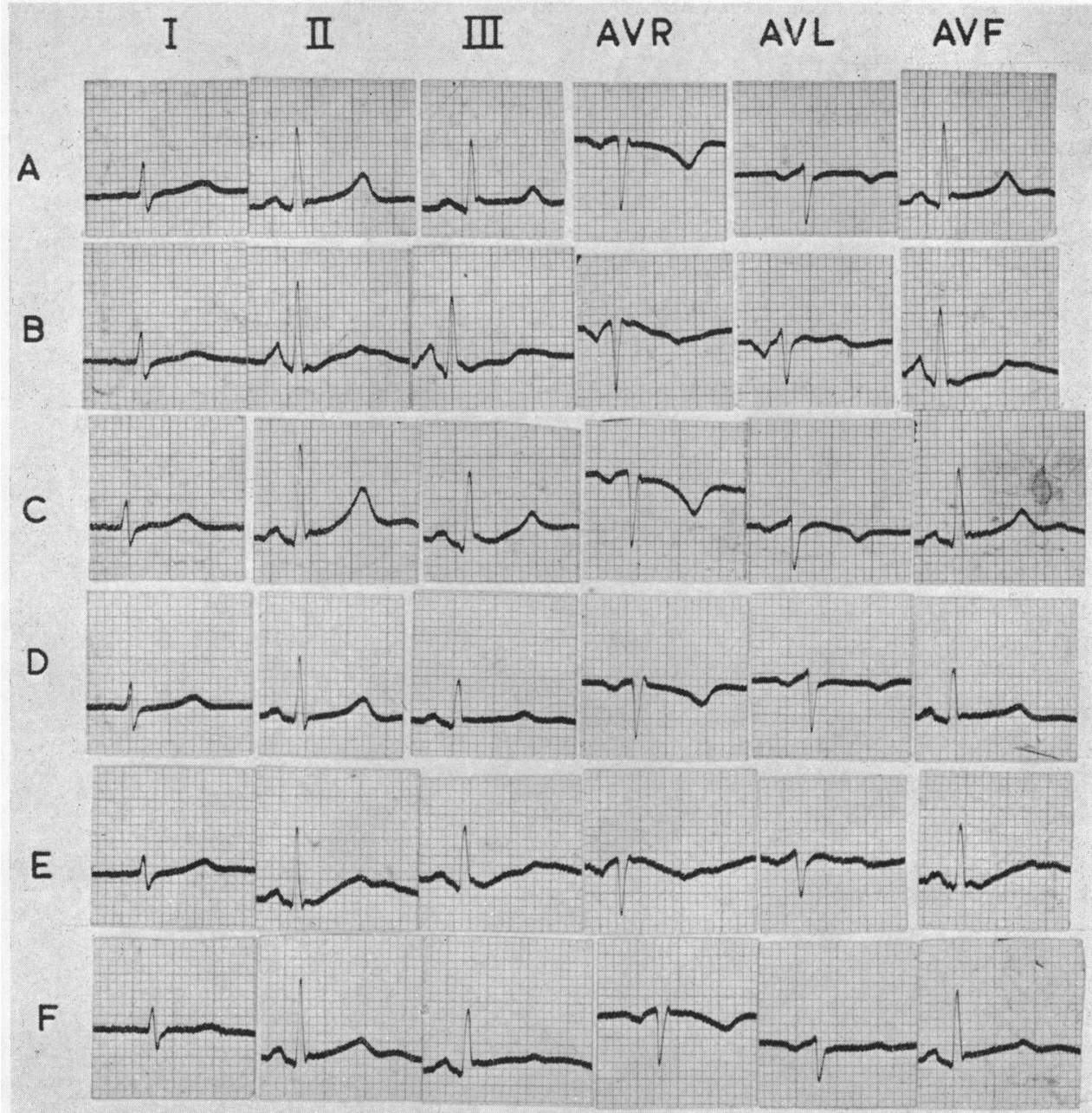

FIG. 2.-The effect of intravenous ergotamine, on the established electrocardiographic changes following a glucose meal, in a patient after a Polya gastrectomy. A, control. B, typical changes present at 14 minutes after glucose meal. At this point ergotamine tartrate $0.5 \mathrm{mg}$. was given intravenously. C, electrocardiographic changes back to control pattern at 26 minutes. D, E. and $F$, show the course of events in same patient without ergotamine. D, control. E, 26 minutes; typical changes still present. F, 50 minutes; recovering.

\section{Possible Causes for Electrocardiographic Changes}

The methods and conditions of the following investigations are described above in the section on Material and Methods unless specially noted below.

\section{Diminished Coronary Blood Flow}

Six patients who had had a Polya gastrectomy were given a rapid intravenous infusion of $500 \mathrm{ml}$. dextran $(6 \%$ in saline) or $500 \mathrm{ml}$. plasma at the time when the E.C.G. changes were beginning to appear following ingestion of the glucose test meal. The volume of plasma (or plasma expander) given was greater than the fall in plasma volume measured at the first experiment, so that a decrease in the circulating blood volume should have been balanced. In only one case was the duration of 
the change shortened compared with that when the test meal was given alone. This suggested that a drop in coronary blood flow was not likely to be the main cause of the E.C.G. changes.

Further evidence against diminished coronary blood flow was found in the effect of an intravenous injection of ergotamine tartrate, $0.5 \mathrm{mg}$. This drug rapidly abolished the E.C.G. changes in nine patients after operation (Fig. 2), in spite of the fact that it tends to constrict coronary vessels (Katz and Linder, 1939).

\section{Lowered Plasma Potassium Levels}

Plasma potassium levels were estimated by means of a flame photometer in 20 patients who developed E.C.G. changes after a glucose meal. No constant trend was found in the test hour, the readings all being within the normal range.

In two patients with hypokalæmia (serum potassium $3.1 \mathrm{~m}$. Eq./1. and $3.5 \mathrm{~m} . \mathrm{Eq} . / 1$.) ergotamine tartrate $0.5 \mathrm{mg}$. intravenously had no effect on the E.C.G. changes, which were typical of potassium deficiency. This was in marked contrast to the rapid reversion to the control pattern in the nine patients who were given ergotamine after the glucose meal (Fig. 2).

\section{Rate of Change in Blood Sugar Level after Glucose Meal}

The blood sugar levels were measured (Hagedorn and Jensen, 1923) in 27 patients after gastrectomy who had E.C.G. tracings. There was no significant difference between the five without E.C.G. changes and the 22 who had such changes.

Four patients who had typical E.C.G. changes after glucose by mouth, were given $0.5 \mathrm{~g}$. glucose per $\mathrm{kg}$. intravenously as a 20 per cent solution. The intravenous glucose produced equivalent blood sugar changes, but no E.C.G. changes.

Three patients were given hypertonic saline $(16 \cdot 3 \mathrm{~g}$. per $100 \mathrm{ml}$, isotonic with the glucose meal) by transnasal jejunal tube, and E.C.G. changes similar to those after a glucose meal were observed (Fig. 3). The pattern was identical in one, slightly less in one, and more fully developed in the third.

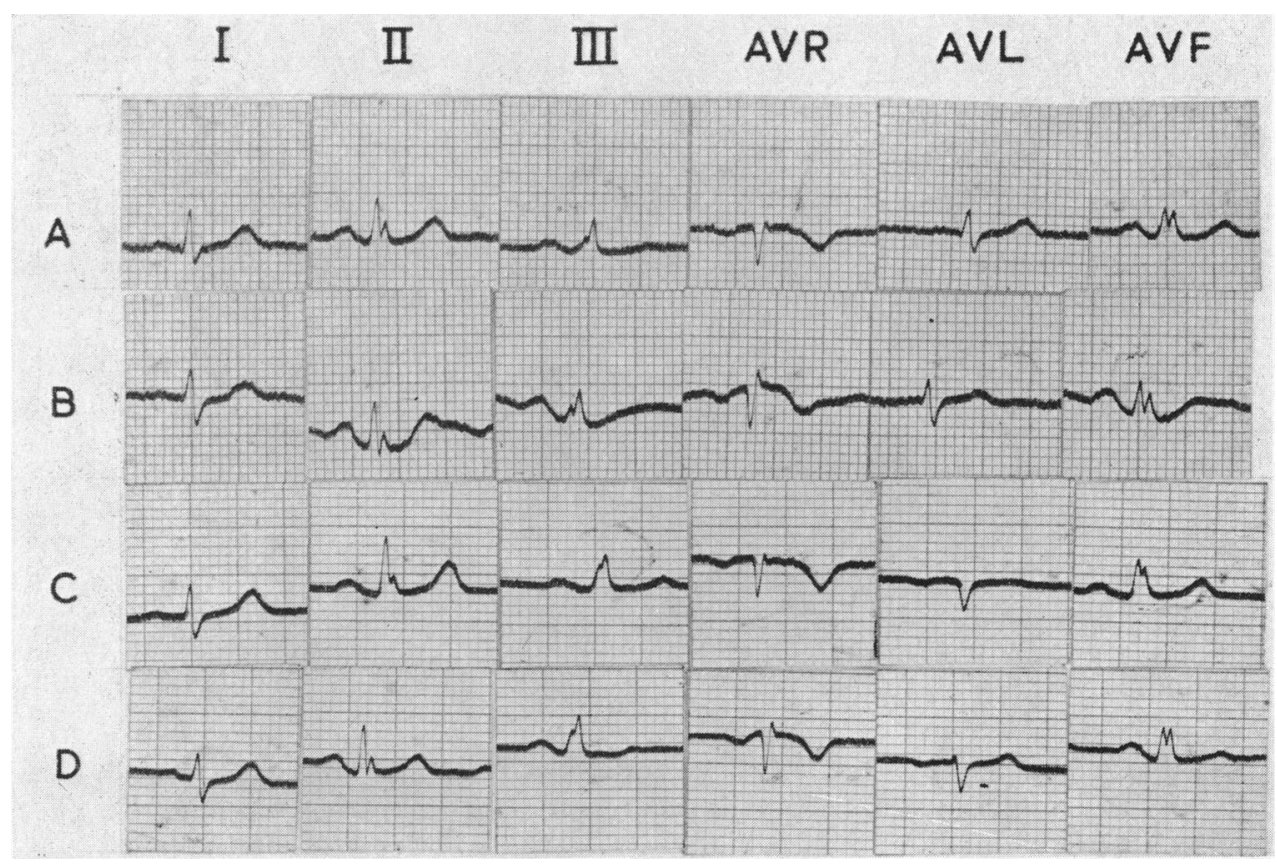

FIG. 3.-Electrocardiographic changes following hypertcnic saline compared with those following a glucose meal in a patient after a Polya gastrectomy. A, control. B, 18 minutes after glucose. C, control. D, 18 minutes after hypertonic saline. 
The above evidence led to the conclusion that the E.C.G. changes were not related to the rise in blood sugar.

\section{Autonomic Imbalance}

Methods designed to alter the balance between the sympathetic and the parasympathetic nervous system were used to study this further.

Effect of Adrenaline. Six patients after Polya gastrectomy were given adrenaline hydrochloride $1 \mathrm{mg}$. by subcutaneous injection and the effect on the E.C.G. compared to that after the glucose meal. The general pattern was seen to be similar (Fig. 4) and the changes were almost identical in three

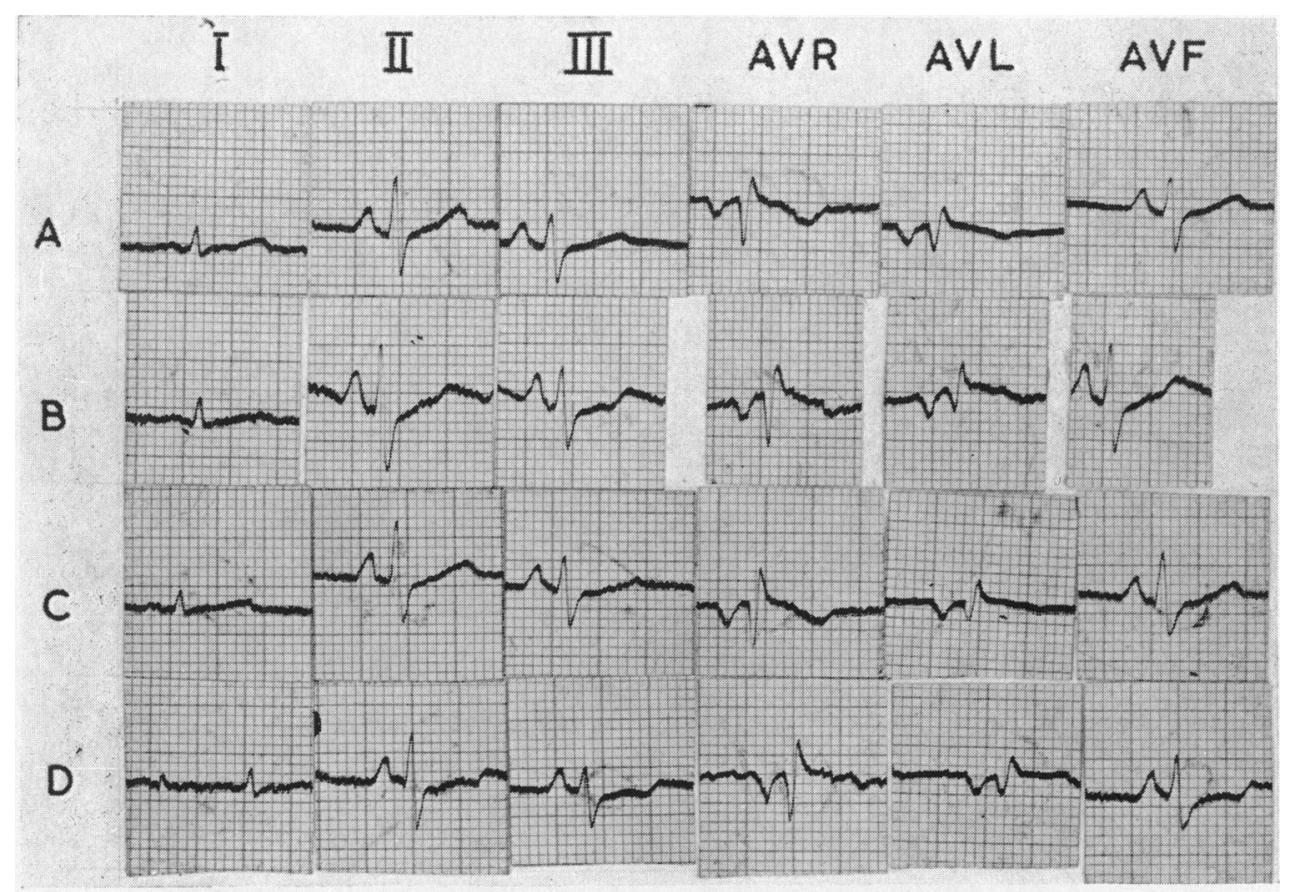

Fig. 4.-Electrocardiographic changes following subcutaneous injection of adrenaline hydrochloride, compared with those following a glucose meal in a patient after a Polya gastrectomy. A, control. $\mathrm{B}, 30$ minutes after glucose meal; C. control. D, 33 minutes after adrenaline, $1 \mathrm{mg}$.

cases. However, following adrenaline $S-T$ changes tended to be more prominent, while $U$ wave changes were less obvious. Furthermore, adrenaline tended to cause arrhythmia; supraventricular extrasystoles were common, and transient inversion of the $P$ wave was seen in three patients.

Effect of Postural Change on the E.C.G. After gastrectomy nine patients who had E.C.G. changes with the glucose meal were tested by taking a control E.C.G. on a horizontal table and then tilting the table to an angle of 75 degrees to the horizontal. Tracings were taken each minute for 10 minutes, after which the patient was returned to the horizontal position.

The pattern of the changes was similar to those seen after glucose (Fig. 5). In six cases the changes were almost identical, and in the remaining three they were less obvious after the postural change than after the glucose meal. These changes began immediately on tilting, and reached a maximum after three to seven minutes. This progression indicated that the changes were not due solely to rotation of the heart.

Effect of Sympatheticolytic Drugs on the E.C.G. Changes Following a Glucose Meal. Phentolamine and phenoxybenzamine hydrochloride (dibenzyline) were given to patients who had had gastrectomy when they were studied by means of the standard glucose meal. Five patients were 


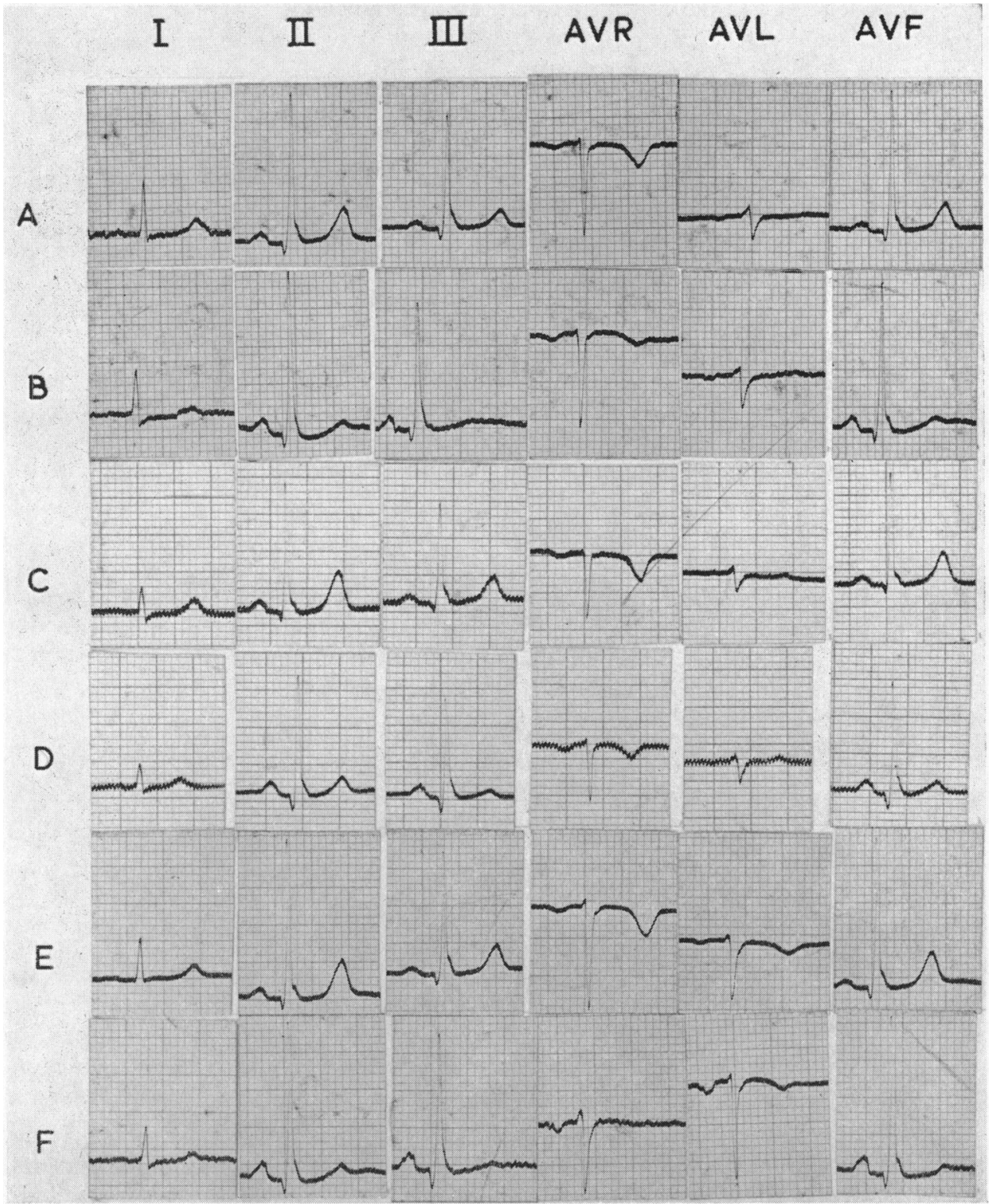

FIG. 5.-Electrocardiographic changes following injection of atropine and after postural change, compared with those following a glucose meal in a patient after a Polya gastrectomy. A, control, sitting. B, 38 minutes after glucose meal. C, control, sitting. D, 15 minutes after atropine gr. $1 / 100$ ( $0.6 \mathrm{mg}$.) intravenously. E, control, lying. D, 5 minutes after tilting table to $75^{\circ}$.

given phentolamine, $10 \mathrm{mg}$. intravenously, and three dibenzyline, 0.7 to $1 \mathrm{mg}$. per $\mathrm{kg}$., by intravenous infusion. In two other patients, a total of $140 \mathrm{mg}$. dibenzyline was given orally in the 12 hours preceding the test meal of glucose. In none of these experiments did the drugs used prevent the changes or accelerate their disappearance; if anything the time for restitution of the E.C.G. changes was prolonged.

Effect of Atropine on the E.C.G. By blocking the vagus, atropine allows overactivity of the 
sympathetic nervous system and tachycardia is one of the results. Atropine also causes $\mathrm{T}$ wave changes in the E.C.G. (Hartwell et al., 1942). Five patients after gastrectomy were given two doses of atropine sulphate gr. 1/100 (0.6 mg.) intravenously at 15 minute intervals under the usual test conditions. Comparison of the maximal changes obtained in this experiment with those following the glucose meal showed that the pattern was similar (Fig. 5) in three patients and less after atropine in two.

Effect of Ergotamine on the E.C.G. Changes Following a Glucose Meal. Ergotamine tartrate $0.5 \mathrm{mg}$. was given by intravenous injection to nine patients after gastrectomy when they had developed E.C.G. changes following a glucose meal. In all nine, the changes were rapidly reversed (Fig. 2) and the E.C.G. returned to the control pattern, except that $U$ waves still tended to be increased. To provide controls for this experiment three patients who had pathologically flattened $T$ waves (two with a left heart strain pattern and one with an old posterior myocardial infarction) were given ergotamine tartrate $0.5 \mathrm{mg}$. intravenously, amyl nitrite being at hand should any deterioration of the tracing occur. No elevation of the $T$ waves occurred, and in one case there was further depression which responded rapidly to the inhalation of amyl nitrite. Scherf and Schlachman (1948b) reported improvement in organic $\mathrm{T}$ wave changes in three out of 13 patients who were given intravenous ergotamine. It can thus be seen that the non-specific improvement is, at best, variable, in contrast to the uniform reversion of the E.C.G. changes after the glucose meal with ergotamine.

A further control was obtained from two patients who were given mephenteramine sulphate $15 \mathrm{mg}$. intravenously at the same time interval as the ergotamine tartrate so that the vasoconstrictor effect of ergotamine might be imitated. No improvement in the E.C.G. appearance resulted.

The dose of ergotamine tartrate given in this investigation is too small to cause a sympathetic block in man, but exercises an effect on the cardiac reflexes by central vagal stimulation (Goodman

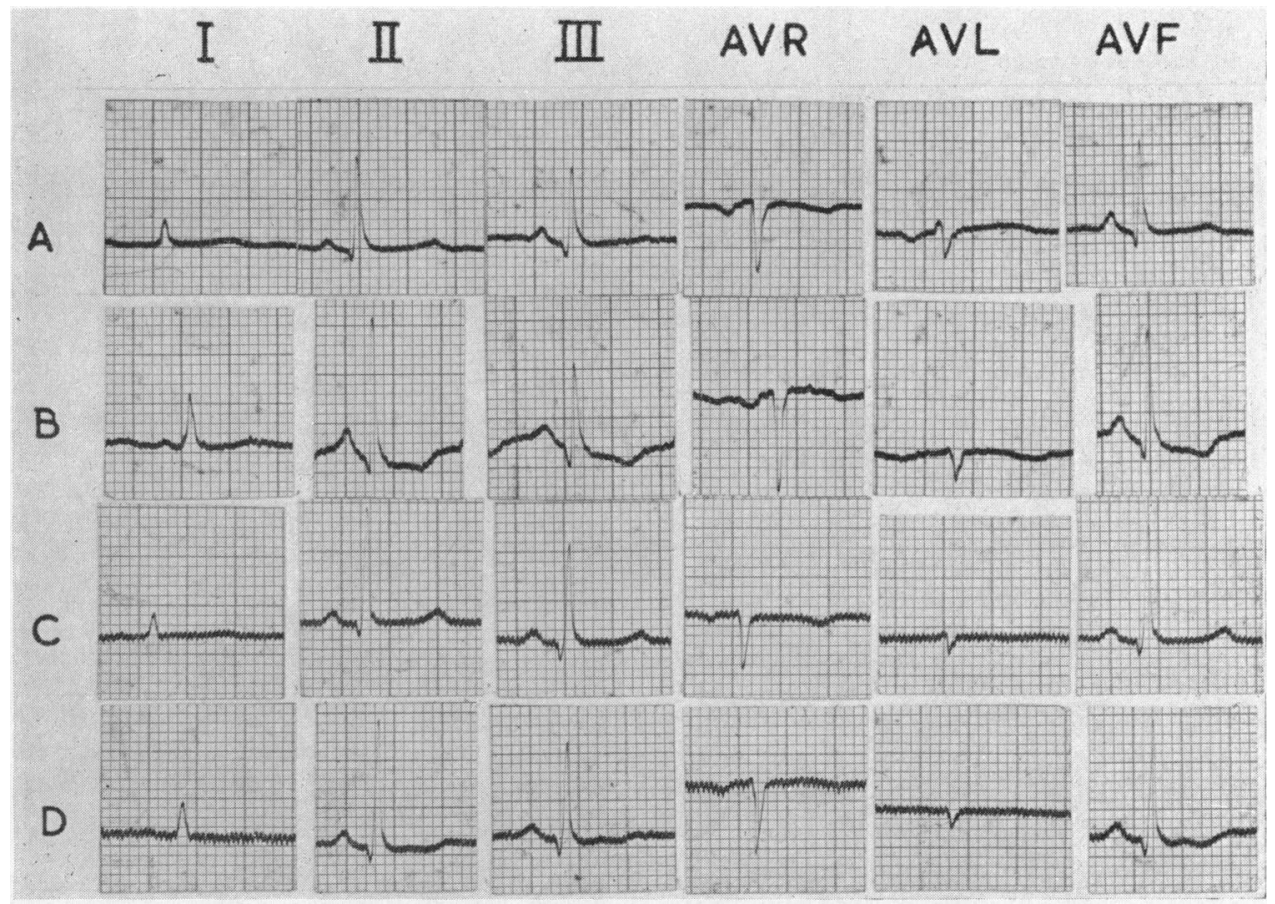

FIG. 6.-Electrocardiographic changes following a glucose meal in a patient after a Polya gastrectomy; first sitting, and later repeated with the patient lying down. A, control, sitting. B, 25 minutes after glucose. C control, lying. D, 25 minutes after glucose. 
and Gilman, 1955). This would be compatible with a relative sympathetic overactivity causing the E.C.G. changes with restoration of the balance by ergotamine.

\section{Effect of Recumbency on the E.C.G. Changes Following a Glucose Meal}

The initiation of these E.C.G. changes is from the alimentary tract, and to attempt to define the mechanism five patients were given a meal of $100 \mathrm{ml} .50$ per cent glucose by transnasal intrajejunal tube in the recumbent position. Some changes in the E.C.G. took place in all patients but they were much less than in the sitting position (Fig. 6).

\section{The Relationship of Heart Rate to the $T$ Wave Changes}

The height of the $\mathrm{T}$ wave was plotted against the corresponding pulse rate. Each was measured from at least four samples in lead II, the analysis being carried out for atropine, postural change,

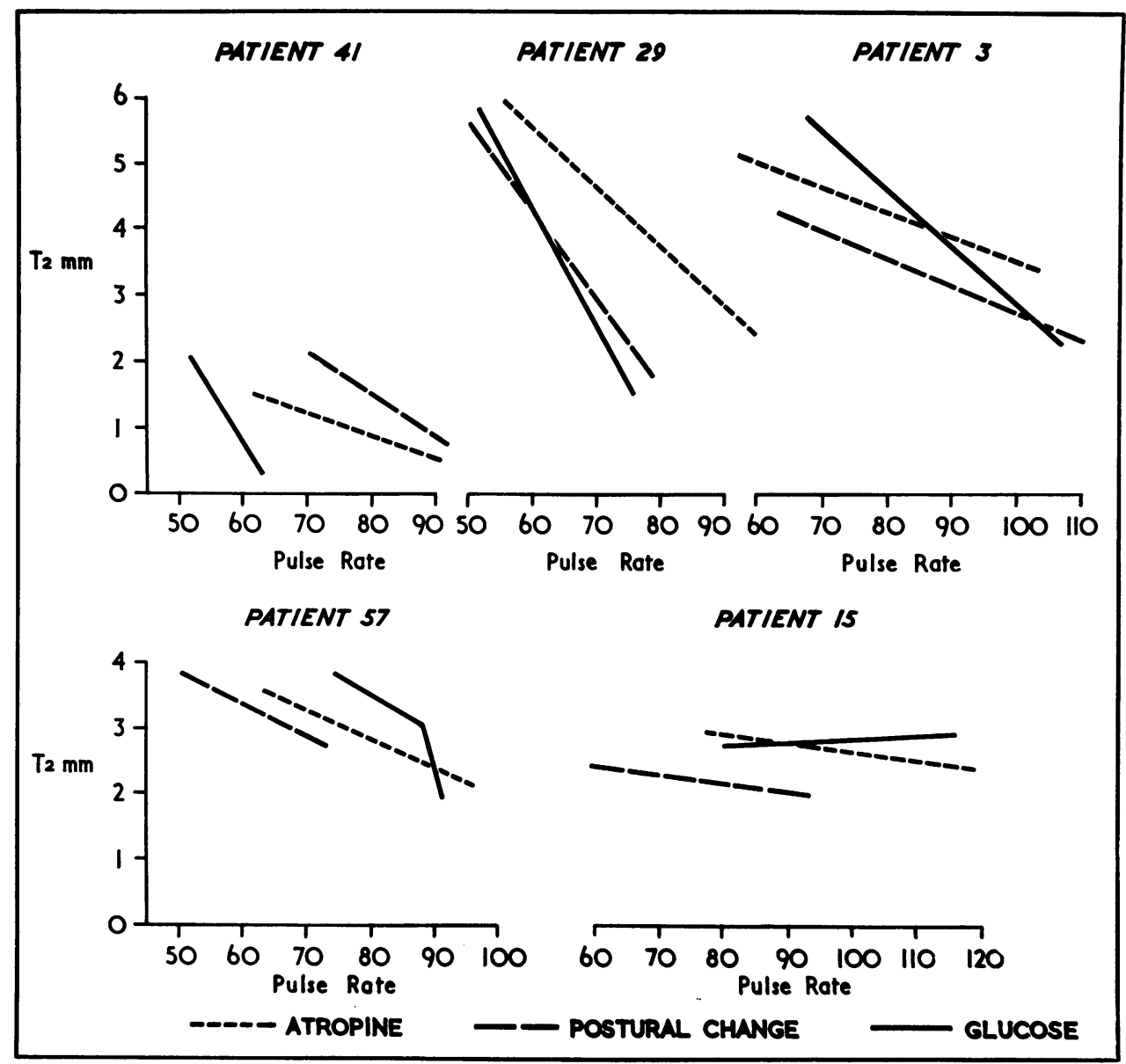

FIG. 7.-The relationship between the pulse rate and the height of the $T$ wave in lead II in five patients in whom the effect of atropine, postural change, and glucose was compared.

and glucose (Fig. 7). Glucose was found to give the steepest slope in four patients. As interpreted by Sjöstrand (1950), this indicated that a hæmodynamic factor, as well as heart rate, influenced the amplitude of the $T$ wave. 


\section{Discussion}

The first part of this study clearly showed that the E.C.G. changes following a glucose meal were similar in the intact stomach and following partial gastrectomy. Gastrectomy merely facilitated and exaggerated the E.C.G. changes indicating that the effect of the operation was to accentuate a physiological process.

The additional experiments pointed to sympathetic stimulation as having a major part in the production of the E.C.G. changes following a glucose meal. The pattern after glucose was similar to that after postural change, after adrenaline and after atropine, and it was reversed by the vagal stimulation of ergotamine. Alteration of posture changes the E.C.G. and the changes have been attributed in part to stimulation of the sympathetic nervous system (Nordenfelt, 1941; Meyerson and Davis, 1942; Scherf and Schlachman, 1948a). Two sympathetic drugs, phentolamine and dibenzyline, produced no improvement, showing that adrenaline release was not the mechanism. That sympathetic stimulation was not the whole explanation was shown by the greater change in some patients following the glucose than after atropine or postural change, and by reversion of the E.C.G. with plasma expander in one case. The fact that some patients had typical E.C.G. changes with an increase in heart rate of only a few beats suggested that tachycardia was not the sole basis of the $T$ wave changes.

When the glucose was given into the jejunum with the patient lying down E.C.G. changes were minimal. It is well known that lying down after a meal prevents vasomotor dumping symptoms and this is generally held to be due to delay in gastric emptying so that the small bowel receives the meal at a slower rate. In this study care has been taken to maintain a similar rate of entry of the glucose into the jejunum when the patient was lying down or sitting in a chair. Despite this, E.C.G. changes were not so marked in recumbency, suggesting that upright posture has some part to play other than hastening gastric emptying. The obvious way in which this operation alters the normal sequence of events after the ingestion of a hypertonic meal is to allow rapid emptying of the gastric remnant which is followed by hypermotility of the small bowel; and this occurs whether dumping is present or not (Duthie and McKellar, 1960). This overactivity of the small bowel has been thought to influence the autonomic nervous system (Hoffman, 1939) and may well be one of the factors initiating the E.C.G. changes. It has been frequently suggested that the hypertonic meal produces an outflow of fluid into the small bowel lumen (Roberts et al., 1954; Amdrup and Jorgensen, 1957; Peddie et al., 1957; Randall, 1958) and the circulatory changes resulting from these two events may assist in producing the E.C.G. changes.

No explanation is provided for the fact that similar E.C.G. changes do not occur in all cases and it can only be postulated that this is due to natural variability in the autonomic neural tone and cardiovascular responses.

\section{SUMMARY}

The electrocardiogram was studied after a standard glucose meal in patients with intact stomach and following gastrectomy. The principal changes were an increase in heart rate, and flattening of the T wave in leads II, III, and aVF. These changes were often associated with depression of the ST-T take-off, and peaking of the P wave, best seen in leads II, III, and aVF. The patterns were similar in the two groups of subjects, but the operation increased the incidence and degree of the changes. In those who had had a gastrectomy, there was no relationship between the E.C.G. changes and the incidence of dumping symptoms, or alterations in other cardiovascular parameters measured.

Further experiments were carried out to evaluate four factors that have been suggested as a basis for the E.C.G. changes; namely, potassium deficiency, rapid glucose absorption, diminished coronary flow, and sympathetic overactivity. The balance of evidence suggested that the last played a major part.

We wish to acknowledge the encouragement and interest of Professor Sir Charles Illingworth during this study and the valuable assistance of Professor E. J. Wayne in the preparation of the paper. Supplies of dibenzyline were kindly provided by Smith, Kline, and French Laboratories, Ltd., Philadelphia, Pa. 


\section{REFERENCES}

Adlersberg, D., and Hammerschlag, E. (1947). Surgery, 21, 720.

Amdrup, E., and Jørgensen, J. B. (1957). Acta chir. scand., 113, 22.

Duthie, H. L., Irvine, W. T., and Kerr, J. W. (1959). Brit. J. Surg., 46, 350.

, and McKellar, N. J. (1960). Brit. J. Radiol., 33, 171.

Gardberg, M., and Olsen, J. (1939). Amer. Heart J., 17, 725.

Goldberger, E. (1953). Unipolar Lead Electrocardiography and Vectorcardiography. 3rd ed. Henry Kimpton, London.

Goodman, L. S., and Gilman, A. (1955). The Pharmacological Basis of Therapeutics. 2nd ed. McMillan Company, New York.

Hagedorn, H. C., and Jensen, B. N. (1923). Biochem. Z., 135, 46.

Hartwell, A. S., Burrett, J. B., Graybiel, A., and White, P. D. (1942). J. clin. Invest., 21, 409.

Hoffman, V. (1939). Münch. med. Wschr., 86, 332.

Katz, L. N., and Linder, E. (1939). J. Amer. med. Ass., 113, 2116.

Meyerson, H. S., and Davies, W. D. (1942). Amer. Heart J., 24, 593.

Mix, C. L. (1922). Surg. Clin. N. Amer., 2, 617.

Nordenfelt, O. (1941). Acta med. scand. Suppl., 119.

Peddie, G. H., Jordan, G. L., and De Bakey, M. E. (1957). Ann. Surg., 146, 892.

Pulvertaft, C. N. (1954). Lancet, 1, 325.

Randall, H. T. (1958). Surg. Clin. N. Amer., 38, 585.

Roberts, K. E., Randall, H. T., Farr, H. W., Kidwell, A. P., McNeer, G. P., and Pack, G. T. (1954). Ann. Surg., 140, 631.

,--- , Bane, H. N., Medwid, A., and Schwartz, M. K. (1955). N.Y. St. J. Med., 55, 2897.

Rochlin, I., and Edwards, W. L. J. (1954). Circulation, 10, 843.

Rosen, I. L., and Gardberg, M. (1957a). Amer. Heart J., 53, 494.

- - . (1957b). Amer. Heart J., 53, 711.

Scherf, D., and Schlachman, M. (1948a). Proc. Soc. exp. Biol. (N.Y.), 68, 150.

- , (1948b). Amer. J. med. Sci., 216, 673.

Simonson, E., Alexander, H., Henschel, A., and Keys, A. (1946). Amer. Heart J., 32, 202.

, and Keys, A. (1950). Circulation, 1, 1000.

Sjöstrand, T. (1950). Acta med. scand., 138, 201.

Smith, W. H. (1951). Lancet, 2, 745.

-, Fraser, R., Staynes, K., and Willcox, J. M. (1953). Quart. J. Med., 22, 381.

Walker, J. N., Roberts, K. E., Medwid, A., and Randall, H. T. (1955). Arch. Surg., 71, 543.

Wilson, F. W., and Finch, R.'(1923). Heart, 10, 275.

Zollinger, R. M., and Hoerr, S. O. (1947). J. Amer. med. Ass., 134, 575. 